\title{
Some features of the triple junction motion in aluminium
}

\author{
S.G. Protasova, V.G.Sursaeva \\ Institute of Solid State Physics, Russian Academy of Sciences, \\ Chernogolovka, Moscow District, 142432 Russia \\ e-mail: sveta@issp.ac.ru, sursaeva@issp.ac.ru
}

Keywords: triple junction, mobility, grain boundaries.

\begin{abstract}
The migration of the systems of tilt grain boundaries with a triple junction $(<100>$, $<110>$ and $<111>$ ) in high-purity aluminium are presented. The experimental results demonstrate that the motion of grain boundary systems with triple junctions in Al at low temperature can be controlled by slowly moving triple junctions. In the high temperature range the triple junctions less affected the motion of the systems and the activation enthalpy was smaller than that at low temperature. The experiments revealed a drastic difference between activation enthalpy of grain boundary and triple junction motion at different temperatures.

The compensation effect (linear dependence of migration activation enthalpy on preexponential mobility factor) at the migration of the systems with triple junction was observed. The compensation temperature was revealed to be close to the temperature for the triple junction mobility.
\end{abstract}

\section{Introduction}

Grain boundaries and triple junctions constitute the dominating elements of polycrystals microstructure. The number of triple junctions in polycrystals is comparable in the order of magnitude with the number of boundaries. Therefore, triple junctions could be chosen equally as structural elements to determine grain growth. The mobility of triple junctions in this case could be derived from a change of the mean displacements of junctions, similar to how grain boundary mobility is extracted from grain growth experiments.

In the polycrystals there are many triple junctions with different crystallographic orientations. Therefore it is very difficult to interpret the regularity of their moving. And so, the motion of the individual triple junction with definite crystallographic parameters was study.

The most important parameter of the motion of this system is the angle $\theta$ in the point where the three boundaries meet (Fig.1). The magnitude of this angle $\theta$ defines the shape of the stationarily moving grain boundary system [1]. The role of the angle $\theta$ becomes clear from the relation between the steady-state value of $\theta$ and the dimensionless criterion $\Lambda$, which describes the influence of the triple junction on the motion of the entire boundary system:

$$
\Lambda=\frac{m_{t j} a}{m_{b}}=\frac{2 \theta}{2 \cos \theta-\sigma_{3} / \sigma}
$$

where $\sigma_{3}$ is the surface tension for the GBIII, $\sigma$ is the surface tension for GBI and GBII, $m_{t j}$ is the triple junction mobility, $m_{b}$ is the grain boundary mobility.

Two limiting cases can be distinguished in this approach:

1. When $A$ is small the angle $\theta$ tends to zero and the steady-state velocity is controlled by the mobility of the junction:

$V=m_{t j} \sigma$

In this case the system movement is controlled by the mobility of the junction (triple junction kinetic) 
2. For $\Lambda>>1$ the junction easily adjusts to the motion of the boundary system, and the angle $\theta$ tends to its equilibrium value $\theta=\arccos \left(\sigma_{3} / 2 \sigma\right)$ and

$V=2 \theta m_{b} \sigma / a$.

In this case the system movement is independent on the mobility of the triple junction and is determined by the boundary mobility (grain boundary kinetic)

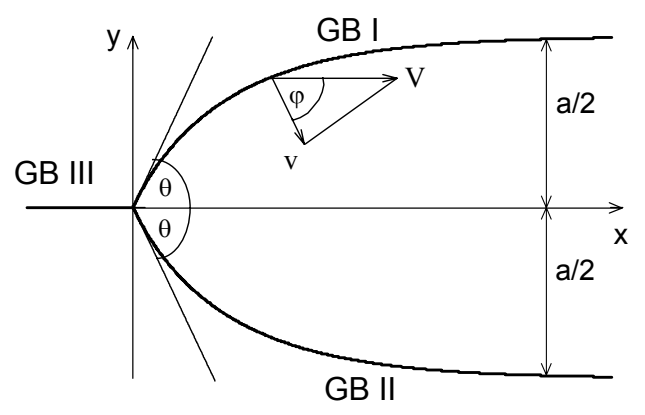

Fig.1. Geometry of the grain boundary system with triple junction during steadystate motion.

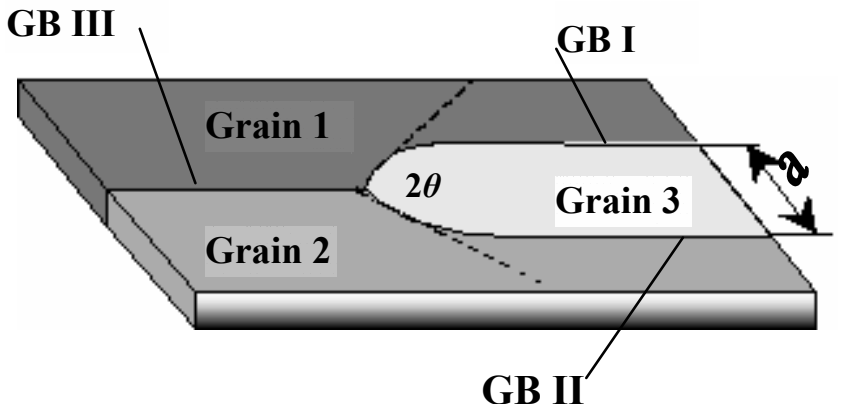

Fig. 2. Scheme of the grain boundary system with a triple junction, $a$ - width of the middle grain; $2 \theta$ - value of the vertex.

\section{Experimental}

The experiments were carried out on tricrystals of high purity $(99,999 \%)$ aluminium with a grain boundary geometry as shown in Fig. 1. The crystallographic characteristics of the studied tricrystals are given in Table 1. The schematic view of the tricrystals is shown in Fig. 2. GB I, GB II, GB III are the tilt grain boundaries with different misorientations angles. The orientations of the three contiguous grains of each sample were determined by the Laue technique.

The tricrystals were grown by directional crystallization in high purity argon atmosphere. Prior to the measurements the samples were electrolytically polished to improve the surface quality and to remove any grain boundary grooves.

Table 1. Crystallography and activation parameters of triple junction mobility in Al.

\begin{tabular}{|c|c|c|c|c|c|c|c|c|}
\hline & GB I & GB II & GB III & $\Delta \mathrm{T},{ }^{\circ} \mathrm{C}$ & $\mathrm{H}_{\mathrm{b}}, \mathrm{eV}$ & $\mathrm{A}_{0 \mathrm{~b}}, \mathrm{~m}^{2} / \mathrm{s}$ & $\mathrm{H}_{\mathrm{tj}}, \mathrm{eV}$ & $\mathrm{A}_{0 \mathrm{tj}}, \mathrm{m}^{2} / \mathrm{s}$ \\
\hline № 1 & $21^{\circ}<111>$ & $18^{\circ}<111>$ & $3^{\circ}<111>$ & $398-479$ & 1,0 & 0,03 & 1,8 & $4,5 \cdot 10^{4}$ \\
\hline № 2 & $20^{\circ}<111>$ & $25^{\circ}<111>$ & $5^{\circ}<111>$ & $380-420$ & & & 2,0 & $1,8 \cdot 10^{6}$ \\
\hline № 3 & $20^{\circ}<111>$ & $10^{\circ}<111>$ & $30^{\circ}<111>$ & $470-510$ & 0,4 & $3,9 \cdot 10^{-6}$ & & \\
\hline № 4 & $22^{\circ}<100>$ & $28^{\circ}<100>$ & $6^{\circ}<100>$ & $460-495$ & & & 3,3 & $1,8 \cdot 10^{13}$ \\
\hline № 5 & $12^{\circ}<100>$ & $25^{\circ}<100>$ & $37^{\circ}<100>$ & $590-620$ & 1,3 & 0,5 & & \\
\hline № 6 & $37^{\circ}<100>$ & $25^{\circ}<100>$ & $12^{\circ}<100>$ & $500-550$ & & & 3,6 & $9,8 \cdot 10^{14}$ \\
\hline № 7 & $12^{\circ}<100>$ & $37^{\circ}<100>$ & $25^{\circ}<100>$ & $520-570$ & 0,9 & $4,7 \cdot 10^{-4}$ & 4,4 & $1,8 \cdot 10^{19}$ \\
\hline № 8 & $27^{\circ}<110>$ & $22^{\circ}<110>$ & $5^{\circ}<110>$ & $469-591$ & 1,4 & 2,3 & 2,7 & $1,3 \cdot 10^{9}$ \\
\hline № 9 & $44^{\circ}<110>$ & $29^{\circ}<110>$ & $15^{\circ}<110>$ & $530-591$ & 1,3 & 0,4 & & \\
\hline
\end{tabular}


For each temperature the velocity $V$, the angle $\theta$, and the width of the shrinking grain $a$ were determined. For this purpose the sample was rapidly cooled down after measurements of the migration rate at each temperature. The position of the grain boundary system and the angle $\theta$ were recorded from the grain boundary grooves. Before subsequent heating to measure the migration rate the specimen was polished to remove the grooves.

\section{Results}

As Fig. 3 shows, the measurement of temperature dependency of $\Lambda$ can be used to separate different regimes of system (grain boundaries and triple junction) motion, i.e. triple junction and grain boundary kinetics.

At relatively low temperatures the $\Lambda$ value is small. Since the criterion $\Lambda$ specifies the ratio of triple junction mobility to grain boundary mobility, then low values of $\Lambda$ mean that the motion of the system in the low-temperature regime can be interpreted as a triple junction controlled motion. While the temperature increasing the $\Lambda$ value becomes higher, which indicates that the system motion is less affected by the triple junction and grain boundary kinetics becomes predominant.
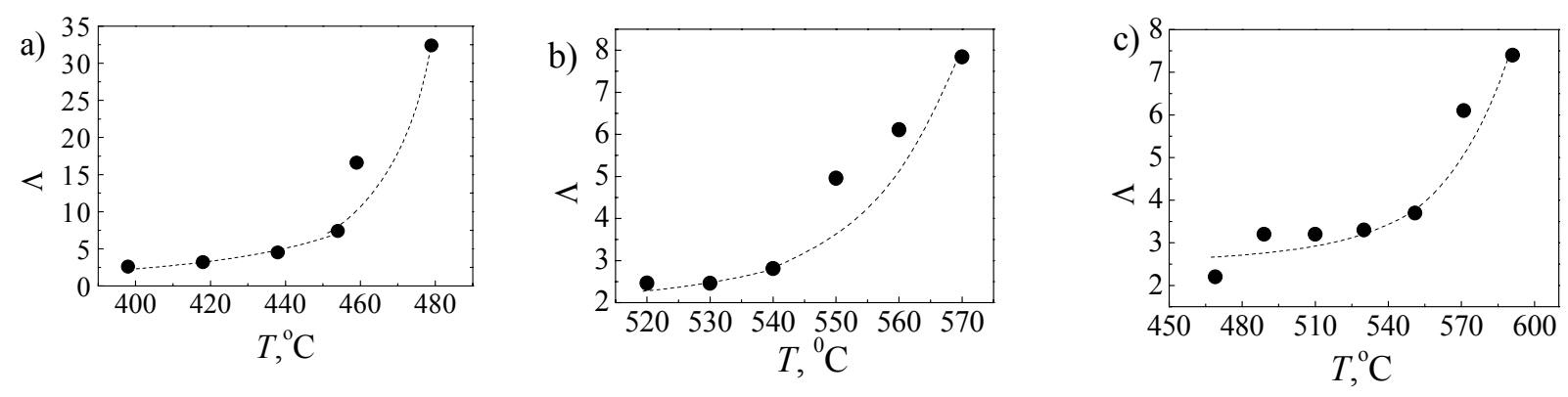

Fig. 3. Temperature dependence of the criterion $\Lambda$ for the triple junctions in samples: a) № 1 [2]; b) № 7; c) № 8 [2].

In compliance with the criterion $\Lambda$ the temperature dependence of triple junction and grain boundary reduced mobility were determined. The results are given in Fig. 4. One can see that two kinetic regimes are observed. The system is obviously controlled by the moving of triple junction at low temperature and by the grain boundary moving at high temperature. According to respective liner behavior in the Arrhenius plot, the activation enthalpy can be determined as indicated in Fig. 4 and Table 1. Apparently the activation enthalpy for the triple junction motion $H_{t j}$ is considerably higher than that for the grain boundary migration $\left(H_{b}\right)$. This rule is remained for another grain boundary with triple junction system, which were studied in the narrow temperature interval. High value of the activation enthalpy for low temperature interval (triple junction kinetic) and small value of the activation enthalpy for high temperature (grain boundary kinetic) are observed. Obviously there is a distinct transition from two temperature intervals: triple junction and grain boundary kinetics also. There exist a temperature (or interval of temperature) at which the transition between two regimes takes place. This temperature is defined by crystallographic parameters of the samples.

The so-called compensation effect was repeatedly observed in various thermally activated processes, but most distinctly in the processes related to interfaces and grain boundaries. The compensation effect is the linear dependence between the activation enthalpy and logarithm of the pre - exponential factor:

$H=\alpha \ln A_{0}+\beta$,

where $\alpha$ and $\beta$ are constants, $H$ the activation enthalpy, $A_{0}$ the pre-exponential factor in the mobility equation $A=A_{0} \exp (-H / k T)$. 

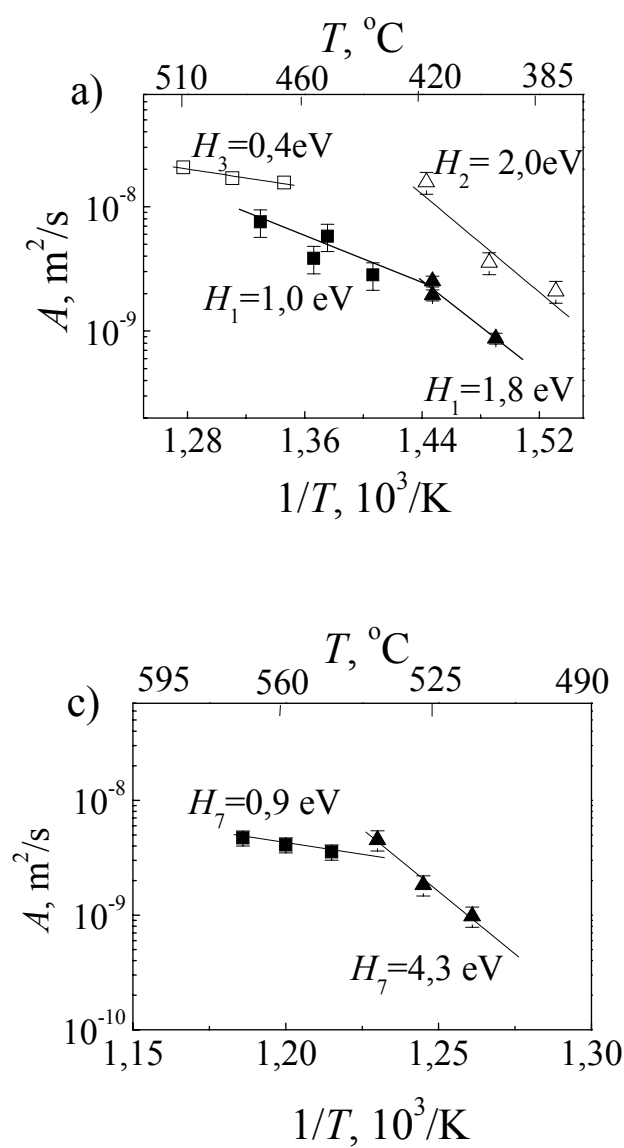

$T,{ }^{\circ} \mathrm{C}$
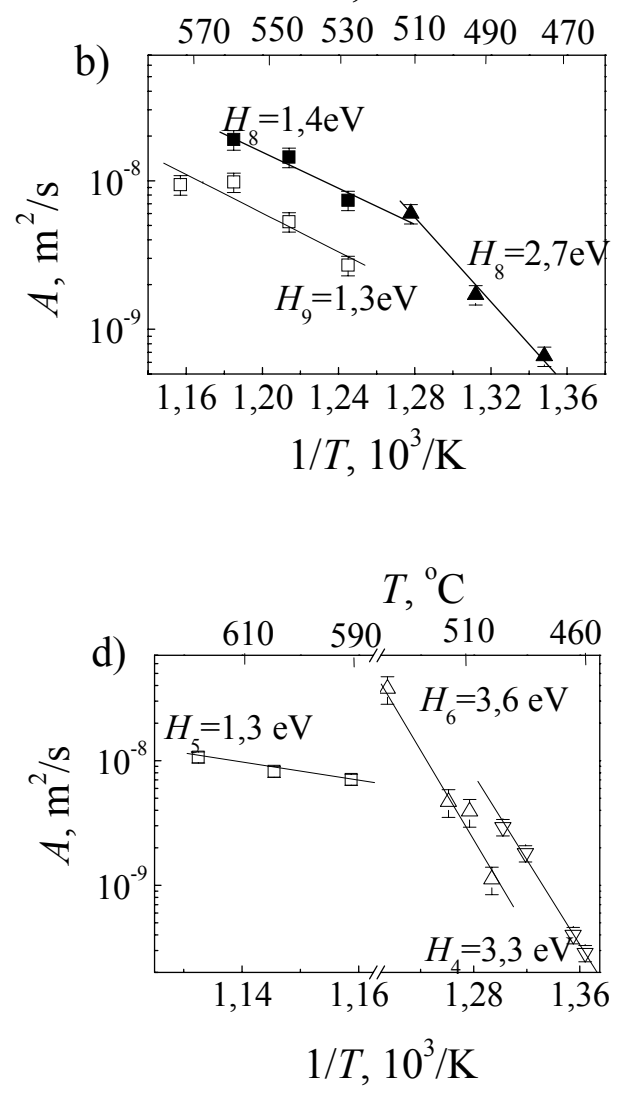

Fig.4. Temperature dependence of the system (grain boundaries and triple junction) mobility in samples: a) <111>: $\mathrm{H}_{1}$-№ 1; $\mathrm{H}_{2}$-№ 2; $\mathrm{H}_{3}$-№ 3; b) <110>: $\mathrm{H}_{8}$-№ 8; $\mathrm{H}_{9}$-№ 9; c), d) <100>: Н7-№ 7; $\mathrm{H}_{4}$-№ 4; $\mathrm{H}_{6}$-№ 6; $\mathrm{H}_{5}$-№ 5 .

The consequence of this effect is the existence of the so - called compensation temperature:

$T_{C}=\alpha / k$.

In concept of [3] the compensation temperature is the equilibrium temperature for a virtual phase transition. The grain boundary with triple junction system has high values of the activation enthalpy and pre - exponential factor and moves faster at temperatures higher than $T_{C}$. In the lower temperature region, the situation is reversed.

The compensation lines for the mobility of the grain boundary with triple junction systems and individual tilt grain boundaries $[4,5,6]$ in $\mathrm{Al}$ are given on the Fig. 5. The value of compensation temperature for the grain boundary with triple junction systems and individual tilt grain boundaries in $\mathrm{Al}$ are given in the Table 2 . The compensation temperature for grain boundary mobility in bicrystals is close to the compensation temperature for the system of grain boundaries with triple junction mobility for two of three crystallografic orientations. Unfortunately the literature data is contradictory and it is difficult to say, why the same regularity is observed.

It is very interesting to compare the temperature of kinetic transition with the compensation temperature (Table 2). One can see that the kinetic transition temperature is close to the compensation temperature for the all systems of grain boundaries with triple junction. One can suppose that the compensation temperature divides the kinetic regimes. Below $T_{C}$ triple junction drags the system motion, and grain boundary kinetics defines the process (triple junction does not drag the system motion) above $T_{C}$. 

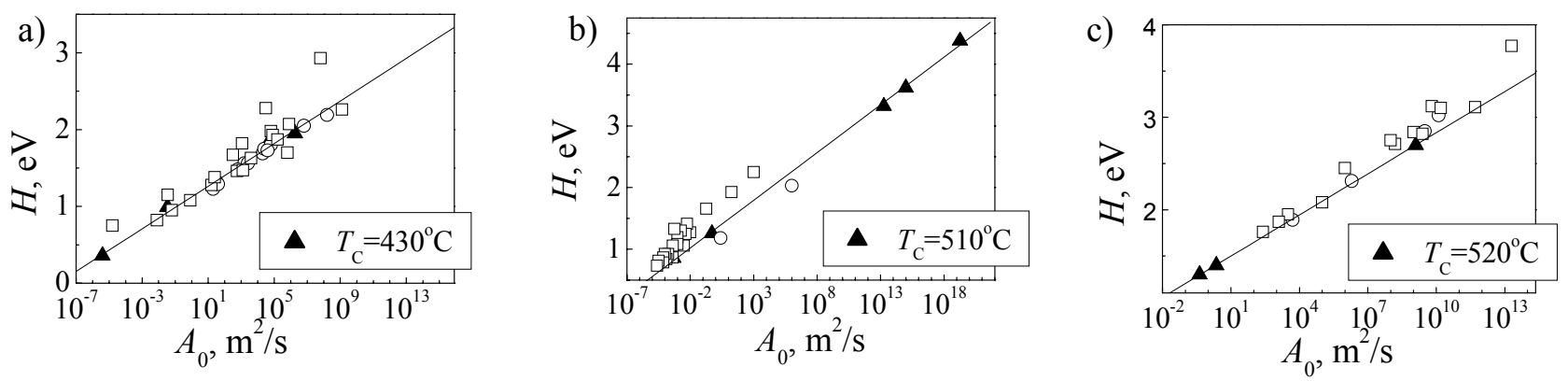

Fig.7. Activation enthalpy $\mathrm{H}$ vs.preexponential factor $\mathrm{A}_{0}$ for: a) $<111>$ : triple junctions in $\mathrm{Al}$ tricrystals- $\mathbf{\Delta}$; grain boundaries in Al bicrystals- $O$ [4], $\square[5]$; b) $<100>$ : triple junctions in $\mathrm{Al}$ tricrystals- $\boldsymbol{\Delta}$; grain boundaries in Al bicrystals- $O$ [6], $\square[5]$; c) $<110>$ : triple junctions in Al tricrystals - $\boldsymbol{\Delta}$; grain boundaries in Al bicrystals- $\bigcirc[5], \square[4]$.

Table 2. Transition temperature $\left(T_{\text {transition }}\right)$ and compensation temperature $\left(T_{\text {compensation }}\right)$ for Al biand tricrystals.

\begin{tabular}{|c|c|c|c|c|}
\hline \multirow{2}{*}{} & \multicolumn{3}{|c|}{$T_{\text {compensation }} / T_{\text {melting }}$} & Triple junctions in Al tricrystals \\
\cline { 2 - 5 } & \multicolumn{3}{|c|}{} & $T_{\text {transition }} / T_{\text {melting }}$ \\
\hline$<100>$ & $1,08[6]$ & $0,82[5]$ & 0,84 & 0,85 \\
\hline$<111>$ & $0,77[4]$ & $0,75[5]$ & 0,75 & 0,76 \\
\hline$<110>$ & $0,92[4]$ & $0,93[5]$ & 0,85 & 0,85 \\
\hline
\end{tabular}

\section{Conclusion}

1. Two kinetics regimes are observed during the motion of grain boundary systems with triple junction in Al tricrystals.

2. Triple junction kinetics manifests itself at low temperature while grain boundary kinetics controls the evolution of a system at elevated temperature.

3. Activation enthalpy for triple junction kinetics is definitely higher than for grain boundary one.

4. Compensation effect for migration of grain boundary system with triple junction was observed. The compensation temperature $T_{C}$ was found to be close to the border between triple junction and grain boundary kinetics. 


\section{References}

[1] A.V. Galina, V.E. Fradkov, and L.S. Shvindlerman, Phys.Metal. Metall. 63,165 (1987)

[2] S.G. Protasova, G. Gottstein, D.A. Molodov, V.G. Sursaeva, L.S. Shvindlerman, Acta Mater., 49 (2001), p 2519

[3] G. Gottstein and L.S. Shvindlerman: Grain Boundary Migration in Metals: Thermodynamics, Kinetics, Applications (CRC Press LLC, 1999)

[4] Aristov, V.Yu.,Kopetskii, Ch.V., Shvindlerman, L.S., In Teoretical Fundamentals of Materials Science, Moscow, Nauka, (1981), p. 84

[5] Gottstein, G., Molodov, D.A., Czubayko, U., Shvindlerman, L.S., J. Physique IV, C3, 5 (1995), p. 89

[6] Fridman, E. M., Kopetskii, Ch.V., Shvindlerman, L.S., Sov. Phys. Solid State, 16 (1974), p. 1152 